PROCEEDINGS OF THE

AMERICAN MATHEMATICAL SOCIETY

Volume 129, Number 7 , Pages $2075-2083$

S 0002-9939(00)05790-7

Article electronically published on December 4, 2000

\title{
NONSTANDARD TOPOLOGIES WITH BASES THAT CONSIST ONLY OF STANDARD SETS
}

\author{
NADER VAKIL
}

(Communicated by Carl G. Jockusch, Jr.)

\begin{abstract}
Let $X$ be an infinite set, $D$ a set of pseudo-metrics on $X$, $\Xi \subset{ }^{*} X$, and $\Gamma \subset{ }^{*} D$. If $\rho(a, b)$ is limited (finite) for every $a, b \in \Xi$ and every $\rho \in \Gamma$, then, for each $\rho \in \Gamma$, we can define a pseudo-metric $\tilde{\rho}$ on $\Xi$ by writing $\tilde{\rho}(a, b)=\operatorname{st}(\rho(a, b))$. We investigate the conditions under which the topology induced on $\Xi$ by $\{\tilde{\rho}: \rho \in \Gamma\}$ has a basis consisting only of standard sets. This investigation produces a theory with a variety of applications in functional analysis. For example, a specialization of some of our general results will yield such classical compactness theorems as Schauder's theorem, Mazur's theorem, and Gelfand-Philips's theorem.
\end{abstract}

\section{INTRODUCTION}

Let $X, D, \Xi$ and $\Gamma$ be as in the abstract. Suppose that ${ }^{\circ} \Xi=\left\{x \in X:{ }^{*} x \in\right.$ $\Xi\} \neq \emptyset$ and ${ }^{\circ} \Gamma=\left\{d \in D:{ }^{*} d \in \Gamma\right\} \neq \emptyset$. Let $\rho(a, b)$ be limited (finite) for every $a, b \in \Xi$ and $\rho \in \Gamma$, and let $\tilde{\Gamma}=\{\tilde{\rho}: \rho \in \Gamma\}$ where $\tilde{\rho}(a, b)=\operatorname{st}(\rho(a, b))$. Let $(\Xi, \tilde{\Gamma})$ denote the uniform space whose uniformity is generated by the set $\tilde{\Gamma}$. The topology induced on $\Xi$ by this uniformity will be referred to as $\tilde{\Gamma}$-topology on $\Xi$. We call $\Gamma$ compatible with $\Xi$ if the $\tilde{\Gamma}$-topology on $\Xi$ has a basis that consists only of standard sets. In this paper we investigate the following question.

Question. Under what conditions on the sets $\Xi$ and $\Gamma$ will the set $\Gamma$ be compatible with $\Xi$ ?

Our main results regarding this question are presented in Section (2), where we treat the question in a setting that is suitable for a wide range of applications. In Section (3), after giving several nonstandard compactness criteria for subsets of a Banach space and its dual, we use the theory developed in Section (2) to derive such classical compactness theorems as Schauder's theorem, Mazur's theorem, and Gelfand-Philips's theorem.

The idea of the $\tilde{\Gamma}$-topology introduced above is an extension of the notion of S-topology introduced by Robinson [1] in the context of metric spaces, and further studied by Henson ([2], page 117) in the context of uniform spaces. What was considered in 2] was a special case of $(\Xi, \tilde{\Gamma})$ in which $\Xi={ }^{*} X$ and $\Gamma=\left\{{ }^{*} d: d \in D\right\}$. In this special case, we shall call the $\tilde{\Gamma}$-topology the Stopology although it was not called so in 2]. The question of existence of a basis

Received by the editors June 8, 1999 and, in revised form, November 16, 1999.

2000 Mathematics Subject Classification. Primary 46S20; Secondary 54J05.

Key words and phrases. Nonstandard topologies, Banach space, compactness conditions. 
consisting only of standard sets for the S-topology was not considered by Robinson or by Henson.

Topologies that are generated by a family of standard sets on ${ }^{*} X$ should not be confused with the S-topology as defined in [1] and [2] and generalized to $\tilde{\Gamma}$-topology here. A special case of the former was first introduced in [4], page 47, and called the discrete S-topology. The idea was somewhat extended and further discussed in [3], page 197, where the relation of such topologies to compactifications of $X$ was pointed out. In [6], starting with a topological space $(X, T)$, Salbany and Todorov considered the topology $\tilde{T}$ that is generated on ${ }^{*} X$ by the collection $\left\{{ }^{*} G: G \in T\right\}$, and used this topology to study various classical compactifications of $(X, T)$.

In [7, H. Render, starting with a uniform space $(X, \mathcal{V})$ and its induced topological space $(X, T)$, studied the relation between the $\tilde{T}$ (as defined in the above paragraph) and the S-topology (as defined on the previous page). The result he announced was that: if $(X, \mathcal{V})$ is not zero-dimensional, then there does not exist a base of the S-topology consisting only of standard sets. But this statement is false. As a trivial example, consider the set $X=(0,1)$ with its usual uniformity (i.e., the one which has the sets $U_{\epsilon}=\{\langle x, y\rangle:|x-y|<\epsilon\}, \epsilon \in R^{+}$, for a basis). By definition, the S-topology associated with this uniformity has the sets $S(a, \epsilon)=\left\{b \in{ }^{*} X:\right.$ st $\left.|a-b|<\epsilon\right\}, \quad a \in{ }^{*} X, \epsilon \in R^{+}$, for a basis. It is obvious that the standard sets ${ }^{*} S(x, \epsilon)$ with $x \in X$ and $\epsilon \in R^{+}$also form a basis for the S-topology. This does not, however, happen in an arbitrary uniform space. The reason it happened here was that the set $X=(0,1)$ with its usual uniformity is a totally bounded space. In Corollary (1.3), we show that, given a uniform space $(X, \mathcal{V})$, the S-topology on ${ }^{*} X$ has a basis consisting only of standard sets if and only if $(X, \mathcal{V})$ is totally bounded.

Thus we first address our opening question in the general setting where it is posed. To fix the notation, let $(X, D)$ be a uniform space with its uniformity generated by a set of pseudo-metrics $D$. If, for an element $a \in{ }^{*} X,{ }^{*} d(a, x)$ is limited for all $d \in D$ and all $x \in X$, then we say that $a$ is limited. We denote the set of all limited elements of ${ }^{*} X$ by $\operatorname{ltd}\left({ }^{*} X\right)$. For other nonstandard concepts used in this paper, we refer the reader to [3]. The reference [5] contains all of the standard concepts used in this paper.

Theorem 1.1. Let $\Gamma=\left\{{ }^{*} d: d \in D\right\}$. A subset $A$ of $X$ is totally bounded in $(X, D)$ if and only if ${ }^{*} A \subset \operatorname{ltd}\left({ }^{*} X\right)$ and the $\tilde{\Gamma}$-topology on ${ }^{*} A$ has a basis consisting only of standard sets.

Proof. Recall that $A$ is totally bounded if and only if every element of ${ }^{*} A$ is pre-near standard. Assume that $A$ is totally bounded. Since ${ }^{*} A \subset \operatorname{pns}\left({ }^{*} X\right) \subset$ $\operatorname{ltd}\left({ }^{*} X\right),{ }^{*} d(a, b)$ is limited for every $d \in D$ and every $a, b \in{ }^{*} A$. Furthermore, for all $a \in{ }^{*} A, x \in X, \epsilon \in R^{+}$, and $d \in D$, the sets

$$
\tilde{S}(a ; x, d, \epsilon)=\left\{b \in{ }^{*} A: \operatorname{st}\left(\left|{ }^{*} d(x, b)-{ }^{*} d(x, a)\right|\right)<\epsilon\right\}
$$

are open relative to the $\tilde{\Gamma}$-topology, are standard, and form a subbasis for the $\tilde{\Gamma}$-topology on ${ }^{*} A$. To prove they are open, fix $b \in \tilde{S}(a ; x, d, \epsilon)$. Choose $\delta=\epsilon-\operatorname{st}\left(\left|{ }^{*} d(x, b) \quad-{ }^{*} d(x, a)\right|\right)$. Since $\tilde{S}(b ; d, \delta) \subset \tilde{S}(b ; x, d, \delta)$, we have $\tilde{S}(b ; d, \delta) \subset \tilde{S}(a ; x, d, \epsilon)$. To see that they are standard, note that the expression $\operatorname{st}\left(\left|{ }^{*} d(x, b)-{ }^{*} d(x, a)\right|\right)$ in (1) can be written as $|\hat{a}(x, d)-\hat{b}(x, d)|$, where $\hat{c}: X \times D \rightarrow R \quad\left(c \in \operatorname{ltd}\left({ }^{*} X\right)\right)$ is defined by $\hat{c}(x, d)=\operatorname{st}\left({ }^{*} d(c, x)\right)$. Thus the 
defining formula of $\tilde{S}(a ; x, d, \epsilon)$ is equivalent to one that contains only standard constants. This proves that the set $\tilde{S}(a ; x, d, \epsilon)$ is standard. Next let $\tilde{S}(a ; d, \epsilon)=$ $\left\{b \in{ }^{*} A: \operatorname{st}\left({ }^{*} d(a, b)\right)<\epsilon\right\}$ be a subbasic element of the $\tilde{\Gamma}$-topology of ${ }^{*} A$. Choose $x \in X$ such that $\operatorname{st}\left({ }^{*} d(a, x)\right)<\frac{\epsilon}{3}$. Then it is easily seen that $\tilde{S}\left(a ; x, d, \frac{\epsilon}{3}\right) \subset \tilde{S}(a ; d, \epsilon)$. This proves that the sets defined in (1) form a subbasis for the $\tilde{\Gamma}$-topology on ${ }^{*} A$. The converse is an easy consequence of the definitions.

In the next two corollaries we continue to assume that $\Gamma=\left\{{ }^{*} d: d \in D\right\}$. Since, for subsets of a complete uniform space, total boundedness and relative compactness are equivalent properties, Theorem (1.1) has the following immediate corollary.

Corollary 1.2. Suppose that $(X, D)$ is a complete uniform space. A subset $A$ of $X$ is relatively compact in $(X, D)$ if and only if ${ }^{*} A \subset \operatorname{ltd}\left({ }^{*} X\right)$ and the $\tilde{\Gamma}$-topology on ${ }^{*} A$ has a basis consisting only of standard sets.

Corollary 1.3. Suppose that $D$ consists only of bounded pseudo-metrics on X. The following assertions are equivalent.

(i) The space $(X, D)$ is totally bounded.

(ii) The space $\left({ }^{*} X, \tilde{\Gamma}\right)$ has a basis consisting only of standard sets.

(iii) The space $\left({ }^{*} X, \tilde{\Gamma}\right)$ is quasi-compact and has $X$ as a dense subset.

Proof. The implication (i) $\Longrightarrow$ (ii) is an immediate consequence of Theorem (1.1). Assume that (ii) holds, and let $\tilde{\mathcal{B}}$ be a basis of $\left({ }^{*} X, \tilde{\Gamma}\right)$ that consists only of standard sets. Since every nonempty member of $\tilde{\mathcal{B}}$ contains a standard element, $X$ is dense in $\left({ }^{*} X, \tilde{\Gamma}\right)$. The quasi-compactness of $\left({ }^{*} X, \tilde{\Gamma}\right)$ follows from the fact if $\tilde{\mathcal{C}}$ is a nonempty subfamily of $\left\{C:{ }^{*} X \backslash C \in \tilde{\mathcal{B}}\right\}$ with the finite intersection property, then $\bigcap \tilde{\mathcal{C}} \neq \emptyset$. For the implication (iii) $\Longrightarrow(\mathrm{i})$, we need only observe that the denseness of $X$ in $\left({ }^{*} X, \tilde{\Gamma}\right)$ implies that every point in $*(X, D)$ is pre-nearstandard.

\section{MAIN THEOREM}

Corollary (1.2) suggests that the notion of compatibility defined in the opening paragraph of the previous section might give rise to interesting compactness conditions in functional analysis. The goal of the present section is to explore this possibility. Theorem (2.3), below, is the main theorem of this paper. First we need to fix the notation. Unless stated otherwise, throughout this section, $X$ and $Y$ are infinite sets, $\Xi$ is a subset of ${ }^{*} X$ with ${ }^{\circ} \Xi=\left\{x \in X:{ }^{*} x \in \Xi\right\} \neq \emptyset, \Gamma$ is a subset of ${ }^{*} Y$ with ${ }^{\circ} \Gamma=\left\{y \in Y:{ }^{*} y \in \Gamma\right\} \neq \emptyset$, and $\sigma$ is a real-valued function on $X \times Y$.

Using $Y$ and $\sigma$ we define a uniform structure on $X$ that is generated by the set of pseudo-metrics $D_{(Y, \sigma)}=\left\{d_{y}: y \in Y\right\}$, where $d_{y}\left(x_{1}, x_{2}\right)=\left|\sigma\left(x_{1}, y\right)-\sigma\left(x_{2}, y\right)\right|$. Now consider the subset $\left\{{ }^{*} d_{b}: b \in \Gamma\right\}$ of ${ }^{*} D_{(Y, \sigma)}$. Given that ${ }^{*} \sigma(a, b)$ is limited for all $a \in \Xi$ and $b \in \Gamma$, we can define a pseudo-metric $\operatorname{st}\left(\left({ }^{*} d_{b}\right)\left(a_{1}, a_{2}\right)\right)$ on $\Xi$ for each $b \in \Gamma$. Thus we can speak of a $\tilde{\Gamma}_{\sigma}$-topology on $\Xi$ induced by these pseudometrics.

A $\tilde{\Xi}_{\sigma}$-topology on $\Gamma$ can be defined in a similar fashion. This topology, being induced on $\Gamma$ by the family $\tilde{\Xi}_{\sigma}=\left\{\operatorname{st}\left({ }^{*} d_{a}\right): a \in \Xi\right\}$ of pseudo-metrics, has as a 
subbase the family $\mathcal{S}$ consisting of the sets

$$
\tilde{S}_{\Xi}(b ; a, \epsilon)=\left\{b^{\prime} \in \Gamma: \operatorname{st}\left(\left|{ }^{*} \sigma\left(a, b^{\prime}\right)-{ }^{*} \sigma(a, b)\right|\right)<\epsilon\right\},
$$

where $b \in \Gamma, a \in \Xi$, and $\epsilon \in R^{+}$. Note that, in case $\Gamma={ }^{*} G$ for some $G \subset Y$, the members $\tilde{S}_{\Xi}(b ; x, \epsilon)$ of $\mathcal{S}$, where $x \in{ }^{\circ} \Xi$, are standard.

Definition 2.1. Let the notations be as in the first paragraph of this section. Suppose that $G$ is a nonempty subset of $Y$ such that ${ }^{*} G \subset \Gamma$. Suppose also that ${ }^{*} \sigma(a, b)$ is limited for all $a \in \Xi$ and $b \in{ }^{*} G$. For $a \in \Xi, b \in{ }^{*} G$, and $\epsilon \in R^{+}$, let

$$
\tilde{S}_{\Xi}(b ; a, \epsilon)=\left\{b^{\prime} \in{ }^{*} G: \operatorname{st}\left(\left|{ }^{*} \sigma\left(a, b^{\prime}\right)-{ }^{*} \sigma(a, b)\right|\right)<\epsilon\right\} .
$$

The $\tilde{\boldsymbol{\Xi}}_{\boldsymbol{\sigma}}$-topology on ${ }^{*} \boldsymbol{G}$ is the topology that has the sets $\tilde{S}_{\Xi}(b ; a, \epsilon)$ for a subbasis. The set $\boldsymbol{\Xi}$ is called $\boldsymbol{\sigma}$-compatible with ${ }^{*} \boldsymbol{G}$ if the sets $\tilde{S}_{\Xi}(b ; x, \delta)$ as $b$ runs through ${ }^{*} G, x$ through ${ }^{\circ} \Xi$, and $\delta$ through $R^{+}$, form a subbasis for the $\tilde{\Xi}_{\sigma}$-topology on ${ }^{*} G$. We call $\boldsymbol{\Xi} \boldsymbol{\sigma}$-compatible with $\boldsymbol{\Gamma}$ if it is $\sigma$-compatible with ${ }^{*} G$ for every ${ }^{*} G$ that is contained in $\Gamma$.

Given that $H$ is a nonempty subset of $X$ such that ${ }^{*} H \subset \Xi$, the definitions of $\tilde{\boldsymbol{\Gamma}}_{\boldsymbol{\sigma}}$-topology on ${ }^{*} \boldsymbol{H}$ and $\boldsymbol{\sigma}$-compatibility of $\boldsymbol{\Gamma}$ with $\boldsymbol{\Xi}$ are obtained symmetrically from the above by an appropriate interposition of notations.

Notation and Definition 2.2. Suppose that $\Xi$ is a union monad (that is, there exists a family $\Sigma$ of subsets of $X$ such that $\Xi=\bigcup\left\{{ }^{*} H: H \in \Sigma\right\}$ ). Note that in this case ${ }^{\circ} \Xi=\bigcup \Sigma$. By $\Sigma_{p}$ we denote the family of all finite subsets of $\bigcup \Sigma$. The set ${ }^{\circ} \Xi$ should also be regarded as a union monad because we can write ${ }^{\circ} \Xi=\bigcup\left\{{ }^{*} H: H \in \Sigma_{p}\right\}$.

We now use the set $\Xi$ to define an infinitesimal relation on ${ }^{*} Y$ as follows. Two elements $b_{1}$ and $b_{2}$ of ${ }^{*} Y$ are called infinitely close relative to $\boldsymbol{\Xi}$ if

$$
{ }^{*} \sigma\left(a, b_{1}\right) \simeq{ }^{*} \sigma\left(a, b_{2}\right) \text { for all } a \in \Xi .
$$

We shall write $(Y, \sigma, \Sigma)$ to indicate that $Y$ is equipped with the uniformity that is determined by this infinitesimal relation. Similarly, we use the set ${ }^{\circ} \Xi$ to define the following infinitesimal relation on ${ }^{*} Y$. We call two elements $b_{1}$ and $b_{2}$ of ${ }^{*} Y$ infinitely close relative to ${ }^{\circ} \Xi$ if

$$
{ }^{*} \sigma\left(a, b_{1}\right) \simeq{ }^{*} \sigma\left(a, b_{2}\right) \text { for all } a \in{ }^{\circ} \Xi .
$$

We shall write $\left(Y, \sigma, \Sigma_{p}\right)$ to indicate that $Y$ is equipped with the uniformity that is determined by the relation of infinitely close with respect to ${ }^{\circ} \Xi$.

Assuming that $\Gamma$ is also a union monad such that $\Gamma=\bigcup\left\{{ }^{*} G: G \in \Omega\right\}$, where $\Omega$ is a family of subsets of $Y$, and that $\Omega_{p}$ is the family of all finite subsets of $\bigcup \Omega$, the notions of infinitely close relative to $\Gamma$ and to ${ }^{\circ} \Gamma$ and the corresponding uniform spaces $(X, \sigma, \Omega)$ and $\left(X, \sigma, \Omega_{p}\right)$ are defined in a symmetric manner.

We are now ready to present the main theorem of this paper.

Theorem 2.3. The notation satisfies all of the conditions stated in (2.2). Suppose also that ${ }^{*} \sigma(x, b)$ and ${ }^{*} \sigma(a, y)$ are limited for all $x \in{ }^{\circ} \Xi, y \in{ }^{\circ} \Gamma, a \in \Xi$, and $b \in \Gamma$. The following statements are equivalent.

(i) $\Xi$ is $\sigma$-compatible with $\Gamma$.

(ii) $\Xi \subset \operatorname{pns}(*(X, \sigma, \Omega))$.

(iii) $\Gamma \subset \operatorname{pns}(*(Y, \sigma, \Sigma))$. 
(iv) $\Gamma$ is $\sigma$-compatible with $\Xi$.

(v) For every $a \in \Xi$ and $b \in \Gamma,{ }^{*} \sigma(a, b)$ is limited and the restriction of the internal function ${ }^{*} \sigma(\cdot, b)$ to ${ }^{*}\left(H, \sigma, \Omega_{p}\right)$ is $S$-continuous, where $H$ is any subset of $X$ with ${ }^{*} H \subset \Xi$.

(vi) For every $b \in \Gamma$ and $a \in \Xi,{ }^{*} \sigma(a, b)$ is limited and the restriction of the internal function ${ }^{*} \sigma\left(a,,^{\circ}\right)$ to ${ }^{*}\left(G, \sigma, \Sigma_{p}\right)$ is $S$-continuous, where $G$ is any subset of $Y$ with ${ }^{*} G \subset \Gamma$.

Proof. (i) $\Longrightarrow$ (ii). Fix $a_{0} \in \Xi, \quad G_{1}, \ldots, G_{n} \in \Omega$, and $\epsilon \in R^{+}$. Choose $H \in \Sigma$ such that $a_{0} \in{ }^{*} H$. We show there is $x \in H$ such that

$$
\left(\forall b \in{ }^{*} G\right)\left[\left|{ }^{*} \sigma\left(a_{0}, b\right)-{ }^{*} \sigma(x, b)\right|<\epsilon\right],
$$

where $G=\bigcup_{i=1}^{n} G_{i}$. The result follows almost immediately once the statement (2) is proved. By hypothesis, ${ }^{*} \sigma(a, y)$ is limited for all $a \in{ }^{*} H$ and all $y \in G$. Hence, for each $a \in{ }^{*} H$, we can define a function $\hat{a}: G \rightarrow R$ by writing $\hat{a}(y)=\operatorname{st}\left({ }^{*} \sigma(a, y)\right)$. The set

$$
\left\{a \in{ }^{*} H:\left(\forall b \in{ }^{*} G\right)\left[\left|{ }^{*} \hat{a}(b)-{ }^{*} \hat{a}_{0}(b)\right|<\frac{\epsilon}{4}\right]\right\}
$$

is standard and contains $a_{0}$, so it contains an element $x \in H$. From this it follows that

$$
(\forall y \in G)\left[\left|\sigma(x, y)-{ }^{*} \sigma\left(a_{0}, y\right)\right|<\frac{\epsilon}{3}\right]
$$

We are now ready to prove (2). Fix $b \in{ }^{*} G$. Then $b \in{ }^{*} G_{j}$ for some $j \in\{1, \ldots, n\}$. The set $\tilde{U}_{\Xi}\left(b ; a_{0}, x, \frac{\epsilon}{4}\right)$ is open in the $\tilde{\Xi}_{\sigma}$-topology of ${ }^{*} G_{j}$. Hence there is a standard element $\tilde{S}$ of the $\tilde{\Xi}_{\sigma}$-topology on ${ }^{*} G_{j}$ such that $b \in \tilde{S} \subset \tilde{U}_{\Xi}\left(b ; a_{0}, x, \frac{\epsilon}{4}\right)$. Choose a standard element $y$ of $\tilde{S}$. Now $b$ and $y$ belong to $\tilde{U}_{\Xi}\left(b ; a_{0}, x, \frac{\epsilon}{4}\right)$, so we have

$$
\left|{ }^{*} \sigma\left(a_{0}, b\right)-{ }^{*} \sigma\left(a_{0}, y\right)\right|<\frac{\epsilon}{3} \quad \text { and } \quad\left|\sigma(x, y)-{ }^{*} \sigma(x, b)\right|<\frac{\epsilon}{3} .
$$

Since $y \in G$, from (3) and (4), we get

$$
\begin{array}{r}
\left|{ }^{*} \sigma\left(a_{0}, b\right)-{ }^{*} \sigma(x, b)\right| \leq\left|{ }^{*} \sigma\left(a_{0}, b\right)-{ }^{*} \sigma\left(a_{0}, y\right)\right| \\
+\left|{ }^{*} \sigma\left(a_{0}, y\right)-{ }^{*} \sigma(x, y)\right| \\
+\left|{ }^{*} \sigma(x, y)-{ }^{*} \sigma(x, b)\right|<\epsilon .
\end{array}
$$

This completes the proof of $((\mathrm{i}) \Longrightarrow($ ii $))$.

(ii) $\Longrightarrow$ (i). If ${ }^{*} G_{0} \subset \Gamma$, there are $G_{1}, \ldots, G_{n} \in \Omega$ such that $G_{0} \subset \bigcup_{i=1}^{n} G_{i}$ (see [3], Theorem (7.4.4.b), page 182). Hence, for each $a \in \Xi$, by (ii), there is $x \in{ }^{\circ} \Xi$ such that $\left|{ }^{*} \sigma(a, b)-{ }^{*} \sigma(x, b)\right|<1$ for all $b \in{ }^{*} G_{0}$. From this it follows that ${ }^{*} \sigma(a, b)$ is limited for all $b \in{ }^{*} G$ and all $a \in \Xi$.

Next we show that the sets $\tilde{S}_{\Xi}(b ; x, \delta)$, as $b$ runs through ${ }^{*} G_{0}, x$ through ${ }^{\circ} \Xi$, and $\delta$ through $R^{+}$, form a standard subbasis for the $\tilde{\Xi}_{\sigma}$-topology on ${ }^{*} G_{0}$. To see this, for each $b \in{ }^{*} G$, define $\hat{b}:{ }^{\circ} \Xi \rightarrow R$ by $\hat{b}(x)=\operatorname{st}\left({ }^{*} \sigma(x, b)\right)$. Note that the sets $\tilde{S}_{\Xi}(b ; x, \delta)$ can be written as

$$
\tilde{S}_{\Xi}(b ; x, \delta)=\left\{b^{\prime} \in{ }^{*} G:\left|\hat{b}(x)-\hat{b^{\prime}}(x)\right|<\delta\right\} .
$$

Hence these sets are all standard. To see that they form a subbasis for the $\tilde{\Xi}_{\sigma^{-}}$ topology on ${ }^{*} G_{0}$, fix an arbitrary subbasic element $\tilde{S}_{\Xi}\left(b_{0} ; a_{0}, \epsilon\right)$ of the $\tilde{\Xi}_{\sigma^{-}}$ topology on ${ }^{*} G_{0}$. By (ii), there is $x_{0} \in{ }^{\circ} \Xi$ such that $\left|{ }^{*} \sigma\left(a_{0}, b\right)-{ }^{*} \sigma\left(x_{0}, b\right)\right|<\frac{\epsilon}{4}$ for all $b \in{ }^{*} G_{0}$. From this, it is easily seen that $b_{0} \in \tilde{S}_{\Xi}\left(b_{0} ; x_{0}, \frac{\epsilon}{4}\right) \subset \tilde{S}_{\Xi}\left(b_{0} ; a_{0}, \epsilon\right)$. 
(ii) $\Longrightarrow$ (iii). Fix $b_{0} \in \Gamma, \epsilon \in R^{+}$, and $H_{1}, \ldots, H_{n} \in \Sigma$. Let $H=\bigcup_{i=1}^{n} H_{i}$ and choose $G \in \Omega$ such that $b_{0} \in{ }^{*} G$. By an argument similar to that given for (3) above, there is an element $y \in G$ such that

$$
(\forall x \in H)\left[\left|\sigma(x, y)-{ }^{*} \sigma\left(x, b_{0}\right)\right|<\frac{\epsilon}{3}\right] .
$$

We need to show that $\left|{ }^{*} \sigma(a, y)-{ }^{*} \sigma\left(a, b_{0}\right)\right|<\epsilon$ for all $a \in{ }^{*} H$. Fix $a \in{ }^{*} H$. By (ii), there is $x \in H$ such that $\left|{ }^{*} \sigma(a, b)-{ }^{*} \sigma(x, b)\right|<\frac{\epsilon}{3}$ for all $b \in{ }^{*} G$. In particular, we have

$$
\left|{ }^{*} \sigma(a, y)-{ }^{*} \sigma(x, y)\right|<\frac{\epsilon}{3} \quad \text { and } \quad\left|{ }^{*} \sigma\left(a, b_{0}\right)-{ }^{*} \sigma\left(x, b_{0}\right)\right|<\frac{\epsilon}{3} .
$$

From (5) and (6), we get

$$
\begin{aligned}
\left|{ }^{*} \sigma(a, y)-{ }^{*} \sigma\left(a, b_{0}\right)\right| \leq\left|{ }^{*} \sigma(a, y)-{ }^{*} \sigma(x, y)\right| & +\left|{ }^{*} \sigma(x, y)-{ }^{*} \sigma\left(x, b_{0}\right)\right| \\
+ & \left|{ }^{*} \sigma\left(x, b_{0}\right)-{ }^{*} \sigma\left(a, b_{0}\right)\right|<\epsilon .
\end{aligned}
$$

This completes the proof of ( (ii) $\Longrightarrow$ (iii) ). The converse of this implication is proved similarly. Also, by symmetry, the proof of the implication ( (iv) $\Longrightarrow$ (iii) ) is similar to that of ( (i) $\Longrightarrow$ (ii)).

The proofs of the equivalences ( (iv) $\Longleftrightarrow$ (v) ) and ( (i) $\Longleftrightarrow$ (vi)) are trivial. Indeed, we need only recall that ${ }^{*} \sigma(\cdot, b)$ is S-continuous at a point $a_{0} \in{ }^{*}\left(H, \sigma, \Omega_{p}\right)$ if and only if for all $\epsilon \in R^{+}$, there are $y_{1}, \ldots, y_{n} \in{ }^{\circ} \Gamma$ and $\delta \in R^{+}$such that for all $a \in{ }^{*} H$ we can write

$$
\max _{1 \leq i \leq n}\left|{ }^{*} \sigma\left(a, y_{i}\right)-{ }^{*} \sigma\left(a_{0}, y_{i}\right)\right|<\delta \longrightarrow\left|{ }^{*} \sigma(a, b)-{ }^{*} \sigma\left(a_{0}, b\right)\right|<\epsilon .
$$

We observe that, under the conditions of theorem (2.3), $\sigma$-compatibility has turned out to be a symmetric relation between $\Xi$ and $\Gamma$. This prompts us to slightly modify our terminology of Definition (2.1) to better reflect this symmetry. Thus we shall say that $\boldsymbol{\Xi}$ and $\boldsymbol{\Gamma}$ are $\boldsymbol{\sigma}$-compatible if and only if any of the equivalent conditions of Theorem (2.3) holds.

There are several standard conditions that are equivalent to the $\sigma$-compatibility of $\Xi$ and $\Gamma$. For example, under the conditions of Theorem (2.3), as is evident from the statements (ii) and (iii) of this theorem, the sets $\Xi$ and $\Gamma$ are $\sigma$-compatible if and only if either of the following equivalent statements holds:

- Every member $H$ of $\Sigma$ is totally bounded in $(X, \sigma, \Omega)$.

- Every member $G$ of $\Omega$ is totally bounded in $(Y, \sigma, \Sigma)$.

We add two more to these in the next theorem, where the notation is as in (2.2). Also recall that if $\mathcal{F}$ is a filter, then $\mu(\mathcal{F})$ denotes its monad.

Theorem 2.4. The sets $\Xi$ and $\Gamma$ are $\sigma$-compatible if and only if $\sigma$ is bounded on $H \times\{y\}$ and $\{x\} \times G$ for all $H \in \Sigma, G \in \Omega, x \in \bigcup \Sigma$, and $y \in \bigcup \Omega$ and any of the following four equivalent statements holds.

(i) Every member $H$ of $\Sigma$ is totally bounded in $(X, \sigma, \Omega)$.

(ii) Every member $G$ of $\Omega$ is totally bounded in $(Y, \sigma, \Sigma)$.

(iii) For all $H \in \Sigma$ and all filters $\mathcal{F}$ on $H$ if $\mathcal{F}$ is a Cauchy filter relative to $\left(H, \sigma, \Omega_{p}\right)$, then it is also a Cauchy filter relative to $(H, \sigma, \Omega)$.

(iv) For all $G \in \Omega$ and all filters $\mathcal{F}$ on $G$ if $\mathcal{F}$ is a Cauchy filter relative to $\left(G, \sigma, \Sigma_{p}\right)$, then it is also a Cauchy filter relative to $(G, \sigma, \Sigma)$. 
Proof. First note that the boundedness conditions on $\sigma$ stated in this theorem are equivalent to those mentioned in Theorem (2.3). Thus the theorem is already proved for (i) and (ii). The theorem for (iii) is symmetric with that for (iv). We thus prove the theorem only for (iii).

Suppose that $\Xi$ and $\Gamma$ are $\sigma$-compatible. For each $H \in \Sigma$ and $y \in \bigcup \Omega$, the internal set $\left\{n \in{ }^{*} N:\left(\forall a \in{ }^{*} H\right)\left[\left|{ }^{*} \sigma(a, y)\right|<n\right]\right\}$ contains all infinitely large $n$, so it must contain a standard $n$. Hence $\sigma$ is bounded on $H \times\{y\}$. Its boundedness on $\{x\} \times G$ is argued similarly.

To prove (iii), let $W$ and $V$ denote the relations of infinitely close on ${ }^{*} X$ relative to $\Gamma$ and ${ }^{\circ} \Gamma$, respectively (see (2.2)). We claim that if $\mathcal{F}$ is a filter on $H$ that is Cauchy relative to $\left(H, \sigma, \Omega_{p}\right)$, then $\mu(\mathcal{F}) \times \mu(\mathcal{F}) \subset W$. Fix $a, a^{\prime} \in \mu(\mathcal{F})$ and $b \in \Gamma$. Since $\mu(\mathcal{F}) \times \mu(\mathcal{F}) \subset V$, we have ${ }^{*} \sigma(a, y) \simeq{ }^{*} \sigma\left(a^{\prime}, y\right)$ for all $y \in{ }^{\circ} \Gamma$. Since, by (2.3.v), the internal function ${ }^{*} \sigma(\cdot, b)$ is S-continuous on ${ }^{*}\left(H, \sigma, \Omega_{p}\right)$, it follows that ${ }^{*} \sigma(a, b) \simeq{ }^{*} \sigma\left(a^{\prime}, b\right)$. Hence $a$ and $a^{\prime}$ are infinitely close relative to $\Gamma$; that is, the ordered pair $\left\langle a, a^{\prime}\right\rangle$ belongs to $W$ as desired.

For the converse, by Theorem (2.3), it suffices to show that (iii) implies (2.3.ii). Fix $a \in \Xi$. Choose $H \in \Sigma$ such that $a \in{ }^{*} H$. Given $\epsilon \in R^{+}$and $y \in{ }^{\circ} \Gamma$, there is a standard $x \in\left\{a^{\prime} \in{ }^{*} H: \operatorname{st}\left(\left|{ }^{*} \sigma(a, y)-{ }^{*} \sigma\left(a^{\prime}, y\right)\right|\right)<\epsilon\right\}$, since the set is standard and non-empty. Hence $a \in$ pns $^{*}\left(H, \sigma, \Omega_{p}\right)$. So there is a Cauchy filter $\mathcal{F}$ on $\left(H, \sigma, \Omega_{p}\right)$ such that $a \in \mu(\mathcal{F})$ (see [4], Theorem (3.12.2), page 77). By (iii), we have $\mu(\mathcal{F}) \times \mu(\mathcal{F}) \subset W$. Since $a \in \mu(\mathcal{F})$, it follows that $\mu(\mathcal{F})$ is contained in $W[a]$ (the monad of $a$ in ${ }^{*}(H, \sigma, \Omega)$ ), which means that $a \in$ pns $*(H, \sigma, \Omega)$. The proof is finished.

The applications of the above results presented in the next section concern the special case where $\Sigma=\{H\}$ for some $H \subset X$ and $\Omega=\{G\}$ for some $G \subset Y$. It is convenient to state this special case in the following corollary.

Corollary 2.5. Suppose that $\emptyset \neq H \subset X$ and $\emptyset \neq G \subset Y$. Suppose also that ${ }^{*} \sigma(a, y)$ and ${ }^{*} \sigma(x, b)$ are limited for all $x \in H, y \in G, a \in{ }^{*} H$, and $b \in{ }^{*} G$. The following statements are equivalent.

(i) The sets ${ }^{*} H$ and ${ }^{*} G$ are $\sigma$-compatible.

(ii) The set $H$ is totally bounded in $(X, \sigma,\{G\})$.

(iii) The set $G$ is totally bounded in $(Y, \sigma,\{H\})$.

(iv) For every $a \in{ }^{*} H$ and $b \in{ }^{*} G,{ }^{*} \sigma(a, b)$ is limited and the restriction of the internal function ${ }^{*} \sigma(\cdot, b)$ to ${ }^{*}\left(H, \sigma,\{G\}_{p}\right)$ is $S$-continuous.

(v) For every $a \in{ }^{*} H$ and $b \in{ }^{*} G,{ }^{*} \sigma(a, b)$ is limited and the restriction of the internal function $\left.{ }^{*} \sigma(a,)^{*}\right)$ to ${ }^{*}\left(G, \sigma,\{H\}_{p}\right)$ is S-continuous.

(vi) Every filter $\mathcal{F}$ on $H$ that is Cauchy relative to $\left(H, \sigma,\{G\}_{p}\right)$ is also Cauchy relative to $(H, \sigma,\{G\})$.

(vii) Every filter $\mathcal{F}$ on $G$ that is Cauchy relative to $\left(G, \sigma,\{H\}_{p}\right)$ is also Cauchy relative to $(G, \sigma,\{H\})$.

\section{Applications}

In this section, after giving nonstandard compactness criteria for subsets of a Banach space and its dual, we use them to derive a number of classical compactness results of functional analysis.

Let $E$ be a Banach space, with $E^{\prime}$ its (continuous) dual. Suppose that $\sigma: E \times E^{\prime} \rightarrow R$ is given by $\sigma\left(x, x^{\prime}\right)=\left\langle x, x^{\prime}\right\rangle=x^{\prime}(x)$, and that $B_{e}$ and $B_{e^{\prime}}$ are 
the closed unit balls of $E$ and $E^{\prime}$, respectively. Recall that the uniformity on $E$ that is determined by the relation of infinitely close relative to ${ }^{*} B_{e^{\prime}}$ is the same as that determined by the norm of the Banach space $E$. Hence a subset $A$ of $E$ is relatively compact in the Banach space $E$ for the norm topology if and only if it is totally bounded in $\left(E, \sigma,\left\{B_{e^{\prime}}\right\}\right)$. Similarly, a subset $A^{\prime}$ of $E^{\prime}$ is relatively compact in the Banach space $E^{\prime}$ for the norm topology if and only if it is totally bounded in $\left(E^{\prime}, \sigma,\left\{B_{e}\right\}\right)$. From these observations and Corollary (2.5), we get the following nonstandard characterizations of compactness in a Banach space and its dual, which might be regarded as the nonstandard version of Bartle's notion of strong pairing [8].

Theorem 3.1. Let $A \subset E$ and $A^{\prime} \subset E^{\prime}$. We have:

(i) $A$ is relatively compact for the norm topology if and only if ${ }^{*} A$ and ${ }^{*} B_{e^{\prime}}$ are $\sigma$-compatible.

(ii) $A^{\prime}$ is relatively compact for the norm topology if and only if ${ }^{*} A^{\prime}$ and ${ }^{*} B_{e}$ are $\sigma$-compatible.

Next, combining Theorem (3.1) with an idea due to S. Kakutani [9], we give a nonstandard proof of the following theorem of Schauder. First, some notation.

Let $E$ and $F$ be Banach spaces. Define $\sigma: E \times E^{\prime} \rightarrow R$ by $\sigma\left(x, x^{\prime}\right)=\left\langle x, x^{\prime}\right\rangle$ and $\gamma: F \times F^{\prime} \rightarrow R$ by $\gamma\left(y, y^{\prime}\right)=\left\langle y, y^{\prime}\right\rangle$. Let $T: E \rightarrow F$ be a continuous linear operator and let $T^{\prime}: F^{\prime} \rightarrow E^{\prime}$ be its adjoint, so that $\left\langle T x, y^{\prime}\right\rangle=\left\langle x, T^{\prime} y^{\prime}\right\rangle$ for all $x \in E$ and $y^{\prime} \in F^{\prime}$. Finally, let $\varphi_{t}: E \times F^{\prime} \rightarrow R$ be defined by $\varphi_{t}\left(x, y^{\prime}\right)=\left\langle T x, y^{\prime}\right\rangle=\left\langle x, T^{\prime} y^{\prime}\right\rangle$.

Theorem 3.2 (Schauder). The operator $T$ is compact if and only if $T^{\prime}$ is compact.

Proof. By definition, $T$ is compact if and only if $T B_{e}$ is relatively compact in $F$. By Theorem (3.1.i), this is equivalent to the condition that ${ }^{*}\left(T B_{e}\right)$ and ${ }^{*} B_{f^{\prime}}$ are $\gamma$-compatible. Since $\varphi_{t}\left(x, y^{\prime}\right)=\gamma\left(T x, y^{\prime}\right)$, this last condition is equivalent to

$$
{ }^{*} B_{e} \text { and }{ }^{*} B_{f^{\prime}} \text { are } \varphi_{t} \text {-compatible }
$$

Since $\varphi_{t}\left(x, y^{\prime}\right)=\sigma\left(x, T^{\prime} y^{\prime}\right), \quad(7)$ holds if and only if ${ }^{*}\left(T^{\prime} B_{f^{\prime}}\right)$ and ${ }^{*} B_{e}$ are $\sigma$-compatible. By Theorem (3.1.ii), this is equivalent to: $\left(T^{\prime} B_{f^{\prime}}\right)$ is relatively compact in $E^{\prime}$, which means $T^{\prime}$ is compact.

As another application of Corollary (2.5), we give a nonstandard proof of Mazur's theorem. For a different proof, one that is based on a nonstandard version of Grothendieck's completeness theorem, see [3], page 282 .

Theorem 3.3 (Mazur). If $A$ is a norm compact subset of a Banach space $E$, then the closed convex hull $\hat{A}$ of $A$ is also norm compact.

Proof. By Theorem (3.1.i) and the fact that $\hat{A}$ is closed, we need only show that ${ }^{*} \hat{A}$ and ${ }^{*} B_{e^{\prime}}$ are $\sigma$-compatible. For this we use (2.5.iv). Fix $\hat{a} \in{ }^{*} \hat{A}$ and $b \in{ }^{*} B_{e^{\prime}}$. We have $\hat{a}=\sum_{i=1}^{\omega} \alpha_{i} a_{i}$, where $a_{i} \in{ }^{*} A, \alpha_{i} \in{ }^{*} R, \alpha_{i} \geq 0$ and $\sum_{i=1}^{\omega} \alpha_{i}=1$. Since ${ }^{*} A$ and ${ }^{*} B_{e^{\prime}}$ are $\sigma$-compatible, there is $M \in R^{+}$such that $\left|\left\langle a_{i}, b\right\rangle\right|<M$ for all $i \in\{1, \ldots, \omega\}$. Hence $|\langle\hat{a}, b\rangle|<M$. Next we show that the restriction of the internal function ${ }^{*} \sigma(\hat{a}, \cdot)$ to ${ }^{*}\left(B_{e^{\prime}}, \sigma,\{\hat{A}\}_{p}\right)$ is S-continuous. Fix $b, b^{\prime} \in{ }^{*} B_{e^{\prime}}$ and assume that $\left\langle\hat{x}, b-b^{\prime}\right\rangle \simeq 0$ for all $\hat{x} \in \hat{A}$. Then $\left\langle x, b-b^{\prime}\right\rangle \simeq 0$ for all $x \in A$. Since the restriction of the internal function ${ }^{*} \sigma(a, \cdot)$ to ${ }^{*}\left(B_{e^{\prime}}, \sigma,\{A\}_{p}\right)$ 
is S-continuous for all $a \in^{*} A$, it follows that $\left\langle a_{i}, b-b^{\prime}\right\rangle \simeq 0$ for all $i \in\{1, \ldots, \omega\}$. Hence $\left\langle\hat{a}, b-b^{\prime}\right\rangle \simeq 0$, as desired.

Finally, we remark that the equivalence of the statements (2.4.i) and (2.4.iv) may be regarded as an extension of Gelfand-Philips's compactness condition for subsets of a Banach space. According to this condition, a subset $A$ of $E$ is relatively compact if and only if it is bounded and, for every bounded filter $\mathcal{F}$ of subsets of $E^{\prime}$, if $\mathcal{F}$ converges to zero pointwise on $E$, then its convergence is uniform on $A$. The derivation of this condition from Theorem (2.4) consists in taking $\Sigma$ as the family of subsets of $E$ that consists of $A$ and all the singletons $\{x\}$, where $x \in E \backslash A$, and letting $\Omega=\left\{B_{e^{\prime}}\right\}$. Then it takes a straightforward argument to show that Gelfand-Philips's condition on bounded filters on $E^{\prime}$ is equivalent to the condition (2.4.iv) imposes on filters on $B_{e}^{\prime}$. But (2.4.iv) is equivalent to (2.4.i), which means that $A$ is relatively compact.

\section{ACKNOWLEDGEMENT}

This paper was written during the author's sabbatical leave from Western Illinois University. The author is also grateful to the anonymous referee whose suggestions improved the exposition.

\section{REFERENCES}

[1] Abraham Robinson, Non-Standard Analysis, North-Holland Publishing Company, Amsterdam, 1966. MR 34:5680

[2] C. Ward Henson, The Nonstandard Hulls of a Uniform Space, Pacific Journal of Mathematics, vol. 43, No. 1, 1972, pp. 115-137. MR 47:2559

[3] W. A. J. Luxemburg and K. D. Stroyan,, Introduction to the theory of infinitesimals, Academic Press, New York, 1976. MR 58:10429

[4] W. A. J. Luxemburg, A general theory of monads, Applications of model theory to algebra, analysis, and probability (W. A. J. Luxemburg, editor), Holt, Reinhart and Winston, New York, 1969. MR 39:6244]

[5] Stephen Willard, General Topology, Addison-Wesley Publishing Company, Reading, Massachusetts, 1970. MR 41:9173

[6] S. Salbany and T. D. Todorov, Nonstandard Analysis in Topology: Nonstandard and Standard Compactifications, to appear in J. Symb. Logic.

[7] H. Render, Topologies on The Nonstandard Model, Topology with Applications, Bolyai Society, Mathematical Studies, 4, Szekszard(Hungry), 1993. pp. 413-418. MR 97a:54052

[8] R. G. Bartle, On Compactness in Functional Analysis, Transactions of the American Mathematical Society, vol. 79, 1955, pp. 35-57. MR 17:510h

[9] S. Kakutani, A proof of Schauder's theorem, Journal of the Mathematical Society of Japan, vol. 3, No. 1, May 1951, pp. 228-231. MR 13:355e

Department of Mathematics, Western Illinois University, Macomb, Illinois 61455

E-mail address: N-Vakil@wiu.edu 